\title{
Fermented pork fat (Sa-um) and lifestyle risk factors as potential indicators for type 2 diabetes among the Mizo population, Northeast India
}

\author{
Freda Lalrohlui', Souvik Ghatak', John Zohmingthanga ${ }^{2}$, Vanlal Hruaii ${ }^{3}$ and Nachimuthu Senthil Kumar ${ }^{1 *}$ (D)
}

\begin{abstract}
Over the last few decades, Mizoram has shown an increase in cases of type 2 diabetes mellitus; however, no indepth scientific records are available to understand the occurrence of the disease. In this study, 500 patients and 500 healthy controls were recruited to understand the possible influence of their dietary and lifestyle habits in relation with type 2 diabetes mellitus. A multivariate analysis using Cox regression was carried out to find the influence of dietary and lifestyle factors, and an unpaired $t$ test was performed to find the difference in the levels of biochemical tests. Out of 500 diabetic patients, 261 (52.3\%) were males and 239 (47.7\%) were females, and among the control group, 238 (47.7\%) were males and 262 (52.3\%) were females. Fermented pork fat, Sa-um (odds ratio (OR) 18.98), was observed to be a potential risk factor along with tuibur (OR 0.1243) for both males and females. Creatinine level was found to be differentially regulated between the male and female diabetic patients. This is the first report of fermented pork fat and tobacco (in a water form) to be the risk factors for diabetes. The unique traditional foods like Sa-um and local lifestyle habits like tuibur of the Mizo population may trigger the risk for the prevalence of the disease, and this may serve as a model to study other populations with similar traditional practices.
\end{abstract}

Keywords: Mizo population, Type 2 diabetes mellitus, Dietary habits, Sa-um, Tuibur, Biochemical profiles

\section{Introduction}

Type 2 diabetes mellitus (T2DM) is a polygenic disorder which arises mainly when the body cannot utilize insulin effectively. The pancreas secretes insulin hormone that helps to carry glucose from the bloodstream into the cells [1]. Elevation in the blood sugar level may lead to acute and long-term complications. T2DM is a chronic illness that requires self-management and medical care which proves to be a complex issue [2]. Diabetes mellitus has been purposively chosen for analysis on the

\footnotetext{
* Correspondence: nskmzu@gmail.com

'Department of Biotechnology, Mizoram University, Aizawl, Mizoram 796004, India

Full list of author information is available at the end of the article
}

ground that it still constitutes one of the major public health problems worldwide. Around the world, the majority of people with diabetes consists mainly of type 2 diabetes, which is found to be the result of physical inactivity and excess body weight. About 2.2 million deaths in 2012 and 1.6 million deaths in 2015 were observed due to the direct cause of diabetes [3]. According to the International Diabetic Federation (IDF), the prevalence of diabetes constitutes $8.7 \%$ of the population in Southeast Asia where $51.1 \%$ are undiagnosed. In India, about 63 million people had diabetes in 2011 with the age range of 20 to 79 years and are expected to rise by 101 million by 2030 [4]. The lifestyle changes, habitual practices, or unique prolonged habits may lead to an 
increase in the incidence of $\mathrm{T} 2 \mathrm{DM}$, and these factors may also vary among the ethnic groups [5].

The risk of T2DM may be triggered by various dietary factors which may include consumption of a highcalorie diet, beverages, and refined foods [6]. As people get older, the risk of T2DM may also increase, especially after the age of 45 years which may be due to less exercise, loose muscle mass, and increase in body weight [7]. Modernization and westernization of lifestyle and dietary habits in developing countries revealed many changes towards a certain type of genes, which may have an adverse effect genetically and in turn lead to susceptibility to type 2 diabetes in different ethnic groups. People became less involved in physical activity while the dietary intake and consumption of meat increases which are consistently associated with risk for diabetes $[8,9]$.

In a small tribal state like Mizoram, the disease occurrence has been reported to be increasing over time, and on contrary, records found in the health centers, clinics, and hospitals are fragmentary, discontinuous, and incomplete to make meaningful dis-aggregation at lower levels and it is difficult to probe the frequency of occurrence [5]. Mizoram is a hilly mountainous region of the Eastern Himalayas and belongs to one of the eight sister states of the North-eastern region of India, bordered by Bangladesh and Myanmar, and is the second least populous state in the country [10]. Tobacco in different forms is used more frequently and a peculiar habit of consumption of tobacco smoke-infused aqueous water ("tuibur") by the Mizo-Mongloid tribe [11, 12]. The people of Mizoram have the habit of chewing "kuhva" (betel leaf with raw areca nut and excess lime) and consumption of smoked meat or vegetables. Rice is the staple food for Mizo tribal people and is mostly favored for breakfast and dinner, while any other foods such as vegetables and meats are considered as side dishes. Spices tend to be used less when compared with other Indian cuisines [13]. Different types of fresh leafy vegetables such as mustard (antam) and pumpkin (maian) leaves are served, and food varieties such as bamboo shoot (mautuai, rawtuai), fermented lard (Sa-um), fermented soya beans (bekang), and dried fish are preserved through smoking. The foods are mostly prepared and served as boiled, stewed, steamed, smoked, or fermented form. Slight variation and modifications in the processing techniques may occur from place to place for ease of preparation and for quality improvement [14].

These unique lifestyle factors of the Mizo people might play a role in the high incidence of T2DM, and the present study was carried out to understand the influence of demographic and epidemiological factors that may contribute to the risk. The study also focuses on the biochemical profiles and their gender-specific variation in type 2 diabetes.

\section{Materials and method \\ Participants}

Five hundred previously diagnosed T2DM patients receiving care from major diagnostic and health clinic "Genesis Laboratory," Aizawl, Mizoram, and five hundred controls volunteered to participate with consent in this study during January 2016-December 2017. The age range for individuals with T2DM and healthy controls was between 40 and 85 years (mean age range $=65.5$ years) were included in this case-control study. The diabetic patients were all previously diagnosed based on the WHO criteria [15]. The work has been approved by ethical committees of Civil Hospital, Aizawl (B.12018/1/13CH(A)IEC/39 dtd. 23/12/2015), and Human Ethical Committee, Mizoram University (MZU/IHEC/2015/006 dtd. 14/12/2015).

Inclusion criteria for this study included the selection of individuals with T2DM after confirmation by a diabetologist, fasting plasma glucose levels $>120 \mathrm{mg} / \mathrm{dl}$, and post-prandial plasma glucose levels $>200 \mathrm{mg} / \mathrm{dl}$. The healthy control group comprised of non-diabetic subjects without the symptoms of diabetes mellitus and blood glucose levels within normal limits (below $<120$ $\mathrm{mg} / \mathrm{dl}$ ). The individuals with T2DM and healthy controls included were from the Mizo ethnic group. The exclusion criteria for sampling included individuals with T2DM with gestational diabetes, diabetes due to pancreatic disorder, pregnancy, and other types of severe illness and metabolic diseases.

\section{Study design and assessment}

The epidemiological information for this case-control study was collected with the consent of the participants. Information on demographic factors, dietary habits, tobacco, alcohol use, and family history was recorded. Dietary habits like meat, smoked meat, salt intake, and Sa-um were taken into account. Lifestyle habits such as cigarette, sahdah, tuibur, paan, and alcohol consumption were recorded (Table 1). Dietary habits were grouped into three categories, viz., low (1 to 2 days per week), moderate (2 to 3 days per week), and high (more than 3 days per week). Blood sugar was estimated by the glucose oxidase-peroxidase (GOD-POD) method, and cholesterol level was estimated by the enzymatic method using cholesterol oxidase/peroxidase aminophenazone (CHOD-PAP reagent) using Sys200 Biochemistry Analyzer [16-18]. Estimation of creatinine was done by the modified Jaffe's method by using a Sys 200 biochemistry analyzer $[16,19]$. The type of medication taken by the patients was recorded as oral or injection. The history of hypertension was also taken into account. Body mass index (BMI) was calculated as weight $(\mathrm{kg}) /$ height $^{2}$ $\left(\mathrm{m}^{2}\right)$ and used to categorize BMI-measured weight status: underweight (BMI $\leq 18.5$ ), normal (BMI 18.5-24.9), 
Table 1 Significant lifestyle and dietary factors used in the univariate analysis. The univariate analysis was carried out to rule out the factors that are deemed to be important to be further analyzed using the multivariate analysis. The odds ratio between the diabetic patients and healthy controls was compared. The $95 \% \mathrm{Cl}$ value was obtained between the cases and the control with a significant value of $p(<0.05)$

\begin{tabular}{llll}
\hline Factors & Odds ratio & 95\% Cl & $p$ value \\
\hline Gender & 1.024 & $0.799-1.313$ & 0.8493 \\
Age $(>45$ diabetic vs $>45$ years control ) & 0.0893 & $0.0426-0.1871$ & $<0.0001$ \\
Education (diabetic vs control) & 1.113 & $0.833-2.141$ & 1.4235 \\
Income (diabetic vs control) & 1.089 & $0.801-1.475$ & 0.9578 \\
Cigarette consumption & 0.7465 & $0.5940-0.9382$ & $<0.0122$ \\
Alcohol consumption & 0.0117 & $0.0016-0.0844$ & $<0.0001$ \\
Paan consumption & 0.0571 & $0.0348-0.0939$ & $<0.0001$ \\
Sahdah consumption & 1.8776 & $1.5139-2.3286$ & $<0.0001$ \\
Tuibur consumption & 2.0130 & $1.3908-2.9137$ & 0.0002 \\
Sa-um consumption & 2.2667 & $1.7115-3.0019$ & $<0.0001$ \\
Meat consumption & 0.4115 & $0.3344-0.5064$ & $<0.0001$ \\
Smoked meat consumption & 0.0695 & $0.0499-0.0970$ & $<0.0001$ \\
Salt consumption & 0.2882 & $0.2309-0.3597$ & $<0.0001$ \\
\hline
\end{tabular}

overweight (BMI 25.0-29.9), and obese (BMI $\geq 30)[20$, 21].

\section{Statistical analysis}

The various lifestyle and dietary factors were analyzed between individuals with T2DM and healthy controls. The statistical analysis was performed using SPSS 20.0 version (IBM Corp, Armonk, NY) software package. A chi-square test was used to assess the association between demographic factors and type 2 diabetes. A logistic regression analysis was carried out to calculate the influence of lifestyle and dietary factors for T2DM. Factors that were deemed of potential importance in the univariate analysis $(p<0.05)$ were further analyzed in the multivariate analysis using Cox regression [22]. The independent effect of risk factors was investigated in a multivariate model (introducing all variables and terms of interactions) retaining the statistically significant factors which show a confounding effect only. Gender and various lifestyle and food habits were all considered in the regression model as potential confounders to evaluate their association with T2DM. The variables were adjusted with age. A receiver operating characteristic (ROC) curve was plotted to find the specificity and sensitivity for the risk factors which achieved a high odds ratio and also to estimate their potential risk score. An unpaired $t$ test was performed for male and female diabetic patients to find the difference in the levels of biochemical tests.

\section{Results}

The total number of participants was 1000 (500 diabetic and 500 controls). Out of 500 diabetic patients, 261
(52.3\%) were males and $239(47.7 \%)$ were females, whereas among control groups, 238 (47.7\%) were males and $262(52.3 \%)$ were females. From the results of the univariate analysis (Table 1) using the chi-square test, the factors that were considered to be of potential importance like alcohol, paan, sahdah, tuibur, Sa-um, meat, smoked meat, and salt were further analyzed using the multivariate analysis. The risk of type 2 diabetes was higher in patients who consumed Sa-um which is a fermented pork fat (odds ratio (OR) 18.98, 95\% confidence interval (95\% CI) 9.8182-36.6918). This was observed with the adjusted age of 45 years or more for both males and females (OR 0.0893, 95\% CI 0.0419-0.5300). Other lifestyle risk factors include betel leaves with areca nut chewing (OR 0.1006, 95\% CI 0.0537-0.1885), tuibur (OR 0.1243, 95\% CI 0.0530-0.2918), and dietary habits like smoked meat (OR 0.0703, 95\% CI $0.0412-0.1200)$. Consumption of salt in excess may also be a risk for type 2 diabetes (OR 0.2134, 95\% CI 0.1400-0.3251) (Table 2). The area under the ROC curve is 0.947 which is predicted to be a potential hazard score for these factors (Figs. 1 and 2). There was no correlation found between BMI and type 2 diabetes in the Mizo population.

Between the male and female diabetic patients, we observed no difference in biochemical parameters like fasting glucose level $(p=0.9813)$, post-prandial glucose level $(p=0.9148)$, cholesterol $(p=0.5673)$, and HbA1c $(p=0.0839)$. However, creatinine level $(p=$ 0.0382 ) was observed to be differentially regulated between male and female diabetic patients in the Mizo population (Fig. 3). 
Table 2 Significant lifestyle and dietary factors used in the multivariate analysis. The factors that were deemed to be of potential significance from the univariate analysis were further analyzed using the multivariate analysis. The odds ratio between the diabetic patients and healthy controls was compared. The $95 \% \mathrm{Cl}$ value was obtained between the cases and the control with a significant value of $p(<0.05)$

\begin{tabular}{llll}
\hline Factors & Odds ratio & $\mathbf{9 5 \% ~ C l}$ & $p$ value \\
\hline Age in years $(>\mathbf{4 5}$ diabetic vs $>\mathbf{4 5}$ control) & 0.1491 & $0.0419-0.5300$ & 0.0033 \\
Paan consumption & 0.1006 & $0.0537-0.1885$ & $<0.0001$ \\
Tuibur consumption & 0.1243 & $0.0530-0.2918$ & $<0.0001$ \\
Sa-um consumption & 18.9802 & $9.8182-36.6918$ & $<0.0001$ \\
Smoked meat consumption & 0.0703 & $0.0412-0.1200$ & $<0.0001$ \\
Salt consumption & 0.2134 & $0.1400-0.3251$ & $<0.0001$ \\
\hline
\end{tabular}

\section{Discussion}

The objective of the study focuses solely on the role of demographic factors which may contribute to the onset of diabetes in the Mizo population. Although the prevalence of T2D increases with obesity, old age, and family history of diabetes, there is also the involvement of certain genetic factors as well as demographic factors [23]. From our study, we found that the Mizo population has their own unique lifestyle and dietary habits which may attribute to the prevalence of type 2 diabetes in Mizoram. The risk of type 2 diabetes was found to be higher in patients consuming Sa-um which is an animal fat product with caul fat adipose tissue which is prepared semi-dry in bottle gourd (Lagenaria siceraria) and although it exhibits distinct astringency it has no significant organoleptic qualities. Sa-um which is also a derivative of pork fat is one of the traditional foods for the local people although it exemplifies adverse health characteristics due to the presence of high saturated fat/ cholesterol content $[24,25]$. In contribution to Sa-um, smoked meat and excess salt consumption may act as secondary risk factor for type 2 diabetes in Mizoram.
Mizo people still practice a variety of food processing habits that were passed on from their forefathers. Only the smoke and the heat directly affect the meat in the traditional smoking process and the meat is generally not barbecued. High-heat cooking many produce harmful chemicals such as polycyclic aromatic hydrocarbons, heterocyclic aromatic amines, and nitrosamines (from nitrates and nitrites added to meats as a preservative) which may lead to an inflammatory response and interfere with the normal production of insulin [26]. High salt or excess salt intake may lead to insulin resistance which in turn may lead to hyperglycemia. Lifestyle factors like betel nut chewing and tuibur consumption also attribute to the risk of developing type 2 diabetes. Some studies show that betel nut chewers may develop T2DM at an early stage which may peak up later in life around the age of 60-69 years [27]. Tuibur is a tobacco-infused water and tobacco is known to be linked with many diseases and nicotine's ability to affect certain antioxidant enzymes like lipid peroxidase, superoxide dismutase, etc., which in turn may contribute to the development of T2DM [28]. Studies from different populations reported

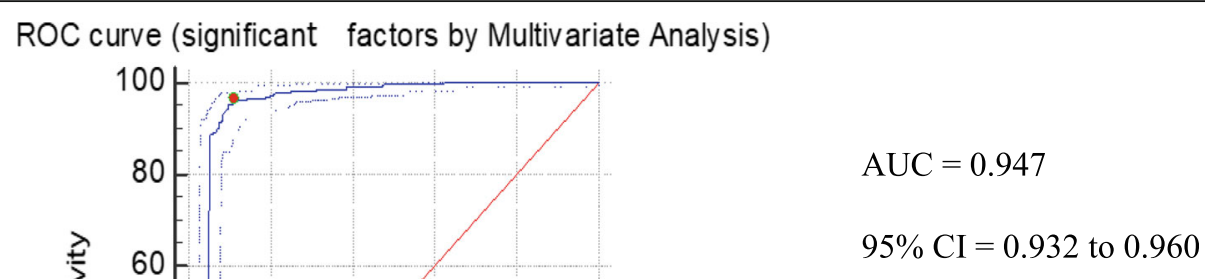

Sensitivity $=96.20$

Specificity $=89.18$ 


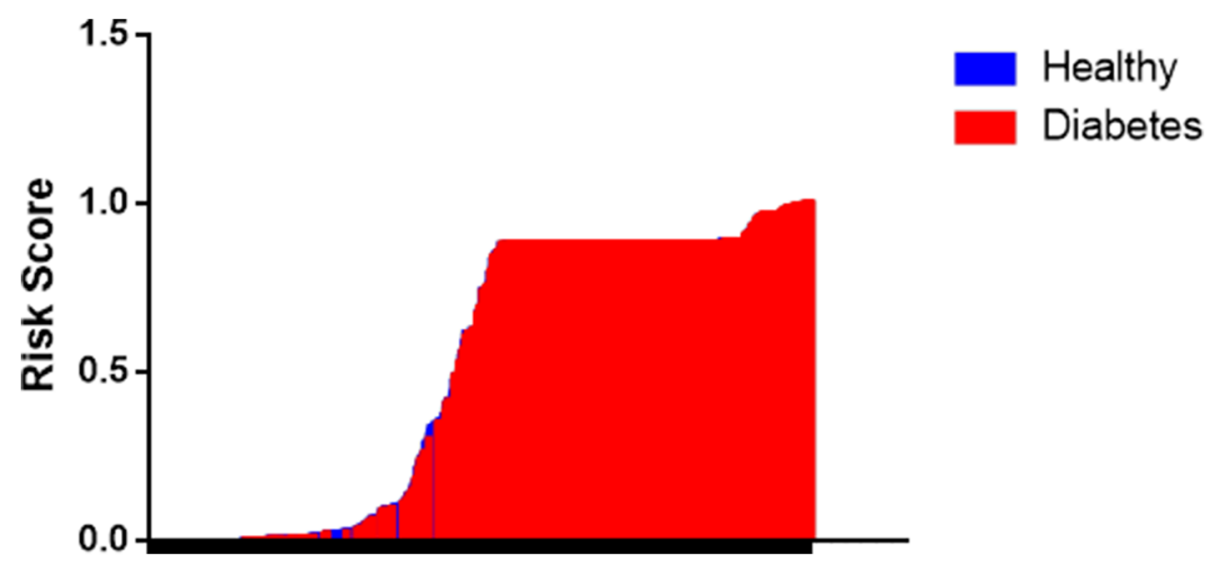

Fig. 2 Estimated risk for the significant demographic factors between diabetic and healthy controls using the multivariate analysis

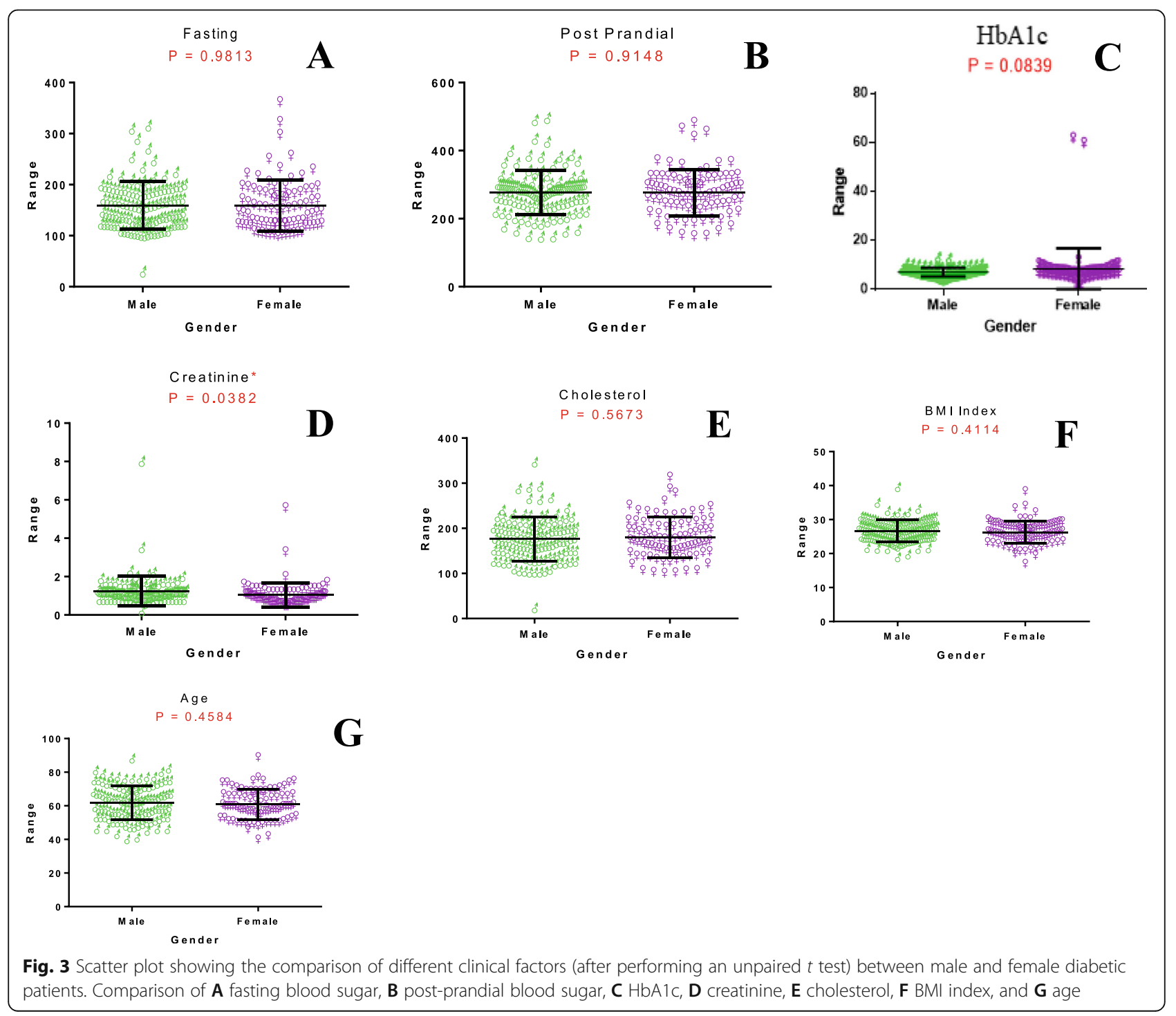


that smoking has an impact on T2DM development [29], as chronic smokers have a higher risk of insulin resistivity [30,31, 32, 33]. Since many risk factors especially those which are modifiable undergo certain changes which may be unfavorable and can in turn cause prevalence of T2DM [34]. However, in our study, we observed no significant relationship between smoking and T2DM in this population [30]. The levels of creatinine may also play a crucial role in depicting the risk for T2DM, especially lower creatinine level, as it is the only substance that is produced by the skeletal muscle mass, which in turn may have a regulatory effect with glucose uptake, thus leading to insulin resistance $[35,36]$. Some of the risk factors that can cause fluctuation in the creatinine levels are cigarette smoking, larger body weight and height, male gender, and old age [37]. Since creatinine level corresponds to muscle mass of the body, the level becomes very likely to increase in males than in females $[38,39]$.

The strength of the present study may include the indepth interview with the participants, large study samples in spite of the small tribal population, standardized measurement for the biochemical profiles in a single laboratory, and adjustment for several confounding factors like meat consumption, alcohol consumption, and family history as well as population-based habits like sahdah (dried tobacco leaves) chewing which were all taken into consideration. To our knowledge, the present study is the first approach to understand the possible relations between unique dietary and lifestyle habits and type 2 diabetes in a tribal population. Although modernization plays an active role in the diet and lifestyle of the people, still diets like Sa-um and smoked food and habits like kuhva and tuibur consumption are still practiced, which may trigger the risk for type 2 diabetes. Thus, the food and lifestyle habits practiced by the people can influence the risk of various diseases, leading to a gradual increase in the acceleration of the disease. A further in-depth study on T2DM involving more samples as well as consumption of various junk foods as per the modern lifestyle habits is required to fully understand the incidence of the disease.

\section{Acknowledgements}

FL would like to acknowledge the Department of Science and Technology, New Delhi, for the DST-INSPIRE fellowship program for the fellowship to the first author (DST/INSPIRE Fellowship/2015/IF150771) and the DBT-Advanced Level State Biotech Hub (BT/04/NE/2009), Mizoram University, for the financial and infrastructural support.

\section{Authors' contributions}

NSK, JZ, and VL planned and proposed the work. FL and SG performed the sampling and experiment. SG and FL performed the statistical analysis. NSK, $\mathrm{JZ}, \mathrm{FL}, \mathrm{VL}$, and SG completed the manuscript. The authors read and approved the final manuscript.

Funding

Not applicable
Availability of data and materials

Please contact the authors for data requests.

\section{Declarations}

\section{Ethics approval and consent to participate}

The study protocol has been approved by the ethical committees of Civil Hospital, Aizawl (B.12018/1/13-CH (A)lEC/39 dtd. 23/12/2015), and Human Ethical Committee, Mizoram University (MZU/IHEC/2015/006 dtd. 14/12/15).

Consent for publication

Not applicable

\section{Competing interests}

The authors declare that they have no competing interests.

\section{Author details}

'Department of Biotechnology, Mizoram University, Aizawl, Mizoram 796004, India. ${ }^{2}$ Department of Pathology, Civil Hospital, Aizawl, Mizoram 796001, India. ${ }^{3}$ Department of Medicine, Zoram Medical College, Aizawl, Mizoram 796005, India.

Received: 23 September 2019 Accepted: 25 June 2021

Published online: 22 July 2021

\section{References}

1. American Diabetes Association. Diagnosis and classification of diabetes mellitus. Diabetes Care. 2007;30(Supplement 1):S42-7. https://doi.org/10.233 7/dc07-S042.

2. American Diabetes Association. Standards of medical care in diabetes. Diabetes Care. 2007;30(Supplement 1):S4-S41. https://doi.org/10.2337/dc07S004.

3. Ramachandran A, Snehalatha C, Shetty AS, Nanditha A. Trends in prevalence of diabetes in Asian countries. World J Diabetes. 2012;3(6):110-7. https://doi. org/10.4239/wjd.v3.66.110.

4. IDF Diabetes Atlas. The global burden. 5th Edition; 2012. p. 23-43.

5. Pulgaron ER, Delamater AM. Obesity and type 2 diabetes in children: epidemiology and treatment. Curr Diabetes Rep. 2014;14(8):508. https://doi. org/10.1007/s11892-014-0508-y.

6. https://shodhganga.inflibnet.ac.in/bitstream/10603/60708/12/12_chapter\%2 04.pdf, http://hdl.handle.net/10603/60708. Retrieved 15 November 2018.

7. Teixeira LE, Nunes $\mathrm{S}$, Teixeira $F$, et al. Regular physical exercise training assists in preventing type 2 diabetes development: focus on its antioxidant and anti-inflammatory properties. Cardiovasc Diabetol. 2011;10(12):14752840.

8. Frank BH. Globalization of diabetes - the role of diet, lifestyle, and genes. Diabetes Care. 2011;34. Diabetes Care. 2011;34 (6):1249-57.

9. Shaw JE, Sicree R. Epidemiology of type 2 diabetes. Contemporary endocrinology: type 2 diabetes mellitus: an evidence-based approach to practical management. Humana Press. 2008; $1^{\text {st }}$ Edition, 2523-3785. https:// www.censusindia.gov.in/2011-prov-results/paper2-vol2/data_files/Mizoram/ Chapter_2.pdf.

10. Barnard N, Levin S, Trapp C. Meat consumption as a risk factor for type 2 diabetes. Nutrients. 2014;6(2):897-910. https://doi.org/10.3390/nu6020897.

11. Registrar General \& Census Commissioner, India. Chapter 2 Size, growth, rate and rural-urban distribution of population in Mizoram (PDF). Retrieved 20 June 2014. https://www.censusindia.gov.in/2011-prov-results/paper2vol2/data_files/Mizoram/Chapter_2.pdf.

12. https://en.wikipedia.org/wiki/Indian_cuisine\#Mizoram. Retrieved 19 November 2018

13. Chakrabarty J, Sharma GD, Tamang JP. Substrate utilization in traditional fermentation technology practiced by tribes of North Cachar Hills district of Assam. Assam University J Sci Tech: Biol Sci. 2009;4:66-72.

14. Lalthanpuii PB, Lalruatfela B. Zoramdinthara, et al. Traditional food processing techniques of the Mizo people of Northeast India. Science Vision. 2017;15(1):39-45.

15. Sreenath M, Nachimuthu SN, Doris Z, et al. Tuibur: tobacco in a bottlecommercial production of tobacco smoke-saturated aqueous concentrate. Addiction. 2018; 113(3):577-80. 
16. Mogre V, Abedandi R, Salifu ZS. Distorted self-perceived weight status and underestimation of weight status in diabetes mellitus type 2 patients. PLoS One. 2014;9:e95165.

17. Shrestha S, Gyawali P, Shrestha R, et al. Serum urea and creatinine in diabetic and non-diabetic subjects. JNAMLS. 2008:9:11-2.

18. Trinder P. Quantitative determination of glucose using GOD-PAP method. Ann Clin Biochem. 1969;6(1):24-7. https://doi.org/10.1177/00045632 6900600108.

19. Bowers LD. Kinetic serum creatinine assays. The role of various factors in determining specificity. Clin Chem. 1980;26(5):551-4. https://doi.org/10.1 093/clinchem/26.5.551.

20. WHO: Definition, diagnosis and classification of diabetes mellitus and its complications, report of a WHO consultation. Geneva, Switzerland:WHO; 1999. Retrieved from http://whqlibdoc.who.int/hq/1999/who_ncd_ncs_99.2. pdf. Accessed Nov 2018

21. WHO: Obesity: preventing and managing the global epidemic, report of a WHO consultation. Geneva, Switzerland:WHO;2000. Retrieved from http:// whqlibdoc.who.int/trs/WHO_TRS_894.pdf?ua=1. Accessed Nov 2018.

22. Devi K, Santhini E, Ramanan D, et al. Mitochondrial ND1 gene mutation analysis in type II diabetes of Karaikudi population. Gene Genom. 2015;38: 37-43.

23. Kumar R, Indrayan A. Receiver operating characteristic curve or ROC curve for medical researchers. Indian Padeatrics. 2011:48(4):277-87.

24. Karen L, Michal A, Jack S. Evaluation of Cox's model and logistic regression for matched case-control data with time-dependent covariates: a simulation study. Statist Med. 2003;22:3781-94.

25. Hooper L, Summerbell CD, Higgins JPT, Thompson RL, Capps NE, Smith GD, et al. Dietary fat intake and prevention of cardiovascular disease: systematic review. BMJ. 2001;322(7289):757-63. https://doi.org/10.1136/bmj.322.7289. 757.

26. Surajit De M, Sambanduram SS, Rajendra BM, et al. Metagenomic analysis and the functional profiles of traditional fermented pork fat 'sa-um' of Northeast India. AMB Express. 2018:8:1-11

27. https://www.hsph.harvard.edu/nutritionsource/2018/03/23/how-meat-iscooked-may-affect-risk-of-type-2-diabetes/. Retrieved 19 November 2018. Accessed Nov 2018.

28. Chin HT. Betel nut chewing and incidence of newly diagnosed type 2 diabetes mellitus in Taiwan. BMC Res Notes. 2010:3:228.

29. Cooper RG. Effect of tobacco smoking on renal function. Indian J Med Res. 2006:124(3):261-8.

30. Willi C, Bodenmann P, Ghali WA, Faris PD, Cornuz J. Active smoking and the risk of type 2 diabetes: a systematic review and meta-analysis. JAMA. 2007; 298(22):2654-64. https://doi.org/10.1001/jama.298.22.2654.

31. Ankit M, Swarkar S, Manoj KD, et al. Risk factors of type 2 diabetes in population of Jammu and Kashmir, India. J Biomed Res. 2013;27(5):372-9.

32. Bamanika SA, Bamanikar AA, Arora A. Study of serum urea and creatinine in diabetic and non-diabetic patients in a tertiary teaching hospital. J Med Res. 2016;2(1):12-5

33. Takeuchi M, Imano H, Muraki I, Shimizu Y, Hayama-Terada M, Kitamura A, et al. Serum creatinine levels and risk of incident type 2 diabetes mellitus or dysglycemia in middle-aged Japanese men: a retrospective cohort study. BmJ Open diab Res Care. 2018;6(1):e000492. https://doi.org/10.1136/ bmjdrc-2017-000492

34. Eric LK, Jacobien CV, Donna S, et al. Factors influencing serum cystatin C levels other than renal function and the impact on renal function measurement. Kidney Int. 2004;65:1416-21.

35. Osamu U, Masataka H, Takeshi M, et al. Age, gender, and body length effects on reference serum creatinine levels determined by an enzymatic method in Japanese children: a multicenter study. Clin Exp Nephrol. 2011; 15:694-9.

36. Xie X, Liu Q, Wu J, Wakui M. Impact of cigarette smoking in type 2 diabetes development. Acta Pharmacol Sin. 2009;30(6):784-7. https://doi.org/10.103 8/aps.2009.49.

37. Facchini FS, Hollenbeck CB, Jeppesen J, Chen YD, Reaven GM. Insulin resistance and cigarette smoking. Lancet. 1992;339(8802):1128-30. https:// doi.org/10.1016/0140-6736(92)90730-Q.

38. Carlsson S, Midthjell K, Grill V. Smoking is associated with an increased risk of type 2 diabetes but a decreased risk of autoimmune diabetes in adults: an 11-year follow-up of incidence of diabetes in the Nord-Trondelag study. Diabetologia. 2004;47(11):1953-6. https://doi.org/10.1007/s00125-004-1554-9.
39. Eliasson B. Cigarette smoking and diabetes. Prog Cardiovasc Dis. 2003;45(5): 405-13. https://doi.org/10.1016/S0033-0620(03)80004-X.

\section{Publisher's Note}

Springer Nature remains neutral with regard to jurisdictional claims in published maps and institutional affiliations.
Ready to submit your research? Choose BMC and benefit from:

- fast, convenient online submission

- thorough peer review by experienced researchers in your field

- rapid publication on acceptance

- support for research data, including large and complex data types

- gold Open Access which fosters wider collaboration and increased citations

- maximum visibility for your research: over $100 \mathrm{M}$ website views per year

At BMC, research is always in progress.

Learn more biomedcentral.com/submissions 